\title{
Opinión del alumnado sobre la implantación del Espacio Europeo de Educación Superior en estudios de Ciencias de la Actividad Física y del Deporte
}

\section{Students' opinion about the implementation of European Higher Education Area in Physical Activity and Sport Sciences studies}

\author{
Javier COTERÓN LÓPEZ, Evelia FRANCO ÁLVAREZ y \\ Javier GIL ARES \\ Universidad Politécnica de Madrid
}

Recibido: Marzo 2012

Aceptado: Abril 2012

\section{Resumen}

El trabajo presenta un análisis descriptivo de la opinión de los estudiantes sobre la realidad universitaria actual en el proceso de cambio promovido por el Plan Bolonia. El estudio se realizó en la facultad de Ciencias de la Actividad Física y del Deporte - INEF de la Universidad Politécnica de Madrid. Se administró un cuestionario validado para la ocasión a una muestra compuesta por 282 estudiantes inmersos en el Plan Bolonia y 203 estudiantes inmersos en el antiguo. Los resultados muestran diferencias en función del plan de estudios cursado. En general, el colectivo de estudiantes de grado presentó valoraciones más positivas acerca de todos los aspectos analizados que el colectivo de estudiantes de licenciatura.

Palabras clave: EEES, opinión estudiantes, enseñanza, organización académica, Plan Bolonia.

\begin{abstract}
The paper presents a descriptive analysis of the students' opinion about the current university reality in the process of change promoted by Bologna Plan. The study was carried out in the Faculty of Physical Activity and Sport - INEF of the Technical University of Madrid. A validated questionnaire was administered to a sample composed by 282 students immersed in the Bologna Plan, and 203 students immersed in the one before. Results show differences according to the curriculum followed. In general terms students belonging to Bologna Plan showed more positive scores than students belonging to the plan before in regard of all analyzed aspects.
\end{abstract}

Keywords: EHEA, students' opinion, teaching, academic organization, Bologna Plan. 
El proceso de convergencia hacia el Espacio Europeo de Educación Superior (EEES) viene protagonizando de forma intensa la vida universitaria durante los últimos años, encontrándose actualmente en plena implantación.

La Declaración de la Sorbona (1998) supone el punto de partida, e irá evolucionando y enriqueciéndose institucionalmente - con la Declaración de Bolonia (1999) y de Praga (2001), y los comunicados de Berlín (2003) y Bergen (2005) - para abordar los nuevos retos educativos dentro de un marco común europeo.

La implantación del EEES en España ha sido tardía: en 2003 se establece el sistema europeo de créditos y el sistema de calificaciones en las titulaciones universitarias de carácter oficial y validez en todo el territorio nacional (Real Decreto 1125/2003); y no es hasta 2007 cuando se fija la ordenación de las enseñanzas universitarias oficiales (Real Decreto 1393/2007).

El cambio promovido con el EEES afecta de modo importante al papel del estudiante. El nuevo sistema se fundamenta en la adquisición de competencias que le permitan un adecuado desempeño de su actividad futura profesional (González y Wagenaar, 2003), haciéndole más partícipe y responsable del proceso de aprendizaje. Para ello, se fomentan a nivel institucional líneas de actuación que permitan el acceso a gran variedad de programas y oportunidades para llevar a cabo estudios multidisciplinares; fomentar la movilidad; facilitar el aprendizaje y perfeccionamiento de idiomas; y promover la utilización de las nuevas tecnologías.

No toda la comunidad estudiantil reaccionó positivamente a la propuesta de cambios sugerida a través de las declaraciones mencionadas anteriormente. Las líneas básicas de protesta se han focalizado contra el encarecimiento de tasas en la universidad, el elitismo de la educación superior, la ausencia de la participación estudiantil en el proceso de cambio, y el aumento de la carga de trabajo en la universidad (Elias, 2010). A pesar de las protestas, el proceso de convergencia ha seguido adelante e, inmersas en el reto que supone la creación del EEES, la mayoría de universidades españolas ha desarrollado diferentes proyectos experimentales y estudios prospectivos para evaluar, entre otros aspectos, el conocimiento y las actitudes de profesores y estudiantes ante las principales novedades del EEES (Castaño, Benito, Portela y Rodríguez, 2007; Font-Mayolas y Masferrer, 2010; Meroño y Ruiz, 2006; Molero, 2007) o la valoración que hacen de los procesos específicos de implantación de los nuevos planes (Castaño, et al., 2007; Mendez, 2008; Meroño y Ruiz, 2006; Montaño, 2008)

La evaluación de estos aspectos adquiere vital importancia en el marco legislativo actual, ya que los títulos universitarios oficiales, para mantener la acreditación como tales y poder seguir siendo impartidos, deberán someterse a un procedimiento de evaluación cada seis años a contar desde la fecha de su inclusión en el Registro de Universidades, Centros y Títulos (Real Decreto 1393/2007).

Además de las reformas producidas en la organización académica, uno de los puntos en los que más se está incidiendo es la renovación metodológica; y así lo han entendido las universidades. Como se señala en las propuestas para la renovación de las metodologías educativas en la universidad (Consejo de Coordinación Universitaria, 
2006), el 75\% de las universidades encuestadas pusieron en marcha planes para mejorar la calidad del aprendizaje incidiendo directamente sobre aspectos metodológicos.

Si tenemos en cuenta que el principal objetivo de este cambio metodológico es la mejora del aprendizaje por parte de los estudiantes, resulta interesante conocer su opinión acerca de la realidad educativa en que están inmersos. Desde esta perspectiva, se han realizado diversos estudios en los últimos años. Castaño et al. (2007) analizaron el impacto de la puesta en marcha de un plan de convergencia, encontrando un aumento de la valoración de la labor del profesorado, si bien existían deficiencias como la coordinación docente y el seguimiento del aprendizaje del estudiante. Iglesia (2011) analizó la valoración del desarrollo competencial en la universidad respecto a las demandas en el entorno laboral, concluyendo que el alumnado percibe una insuficiente formación competencial para su futuro desarrollo profesional. Mallado y Pérez-López (1985) preguntaron a los estudiantes sobre diversos aspectos de la labor docente, encontrando que los más valorados se relacionan con la actividad dentro del aula y la capacidad de innovación. Ruiz y Oliveros (2006) analizaron también la labor docente encontrando entre el alumnado, de forma general, una preferencia por la enseñanza de carácter teórico-práctico que promoviera la participación del alumnado. Sáez (2000) encontró resultados similares al comparar la opinión de estudiantes que eligieron diferentes modelos de enseñanza.

En la Universidad Politécnica de Madrid (UPM), las primeras titulaciones de grado/máster empezaron a impartirse en el curso 2009-2010, ampliándose a la totalidad en el curso siguiente. En los años precedentes, se realizó un esfuerzo, a través de un plan de innovación educativa promovido por el Vicerrectorado de Ordenación Académica, para informar convenientemente a la comunidad educativa, formar al profesorado y diseñar una transición adecuada al nuevo modelo.

El estudio se llevó a cabo en la facultad de Ciencias de la Actividad Física y del Deporte (FCCAFD) de la UPM a finales del curso 2010-2011, como parte de un proyecto de innovación educativa financiado por la universidad. Habiéndose iniciado la implantación de los estudios de grado en el curso 2009-2010, en el momento de su realización coexistían ambos planes de estudios: los dos primeros cursos - grado - se impartían según los nuevos planteamientos; y los tres últimos seguían el plan de estudios - licenciatura - a extinguir. El estudio se lleva a cabo, por tanto, en el ecuador del proceso formativo de la primera generación de estudiantes tras la implantación del EEES; y lo consideramos un momento óptimo porque tanto los estudiantes de grado como los de licenciatura han tenido un contacto directo con los cambios que conlleva el nuevo plan, lo que les ha permitido forjarse su propia opinión al respecto.

El presente trabajo tiene como objetivo fundamental analizar la percepción que los estudiantes de la facultad, los de grado y licenciatura, tienen sobre a) la universidad, b) la organización académica, c) la enseñanza y d) el plan Bolonia; y estudiar posibles diferencias entre ambos colectivos. Como objetivo complementario, se pretende analizar si existen diferencias según el género. 


\section{Método}

\section{Muestra}

La muestra utilizada (ver Tabla 1) ha sido de 485 estudiantes (341 varones y 144 mujeres) de la FCCAFD de la UPM; 282 eran estudiantes de grado en Ciencias del Deporte y 203 de licenciatura en Ciencias de la Actividad Física y del Deporte.

Tabla 1. Distribución de la muestra según el género y la titulación de los estudiantes

\begin{tabular}{llll}
\hline \multicolumn{2}{l}{ Distribución por género } & \multicolumn{2}{l}{ Distribución por Titulación } \\
\hline Hombres & Mujeres & Grado & Licenciatura \\
341 & 144 & 282 & 203 \\
$(70.31 \%)$ & $(29.69 \%)$ & $(58.14 \%)$ & $(41.86 \%)$ \\
\hline
\end{tabular}

La media de edad de los participantes es de 20.25 años, aunque existe una gran variabilidad en la misma $(\mathrm{DT}=5.66)$. Atendiendo al plan de estudios que estén cursando, la edad media de los estudiantes de grado se sitúa en 19.7 años y la de los de licenciatura en 21 años.

En cuanto al género de los estudiantes encuestados, el $70 \%$ de la muestra corresponde a chicos y el $30 \%$ a chicas. Esta proporción coincide con la distribución por género que suele concurrir tradicionalmente en los estudios universitarios de CCAFD según distintas investigaciones (ANECA, 2005)

\section{Diseño y Procedimiento}

El estudio es de carácter transversal, utilizando para el análisis de datos una metodología descriptiva.

El procedimiento para su desarrollo se planteó en dos fases: una primera de elaboración y validación de un cuestionario; y una segunda de recogida, análisis de datos y elaboración de conclusiones.

El cuestionario elaborado consta de 26 ítems, agrupados en torno a cuatro ámbitos: aspectos generales de los estudios; organización académica de la universidad y la facultad; enseñanza y metodología docente; y opinión sobre el Plan Bolonia. En cada ítem, formulado como una afirmación, se pide a los sujetos que indiquen, en una escala tipo Líkert con valores de 1 al 5 (siendo 1 "totalmente en desacuerdo" o "muy negativo"; y 5, "totalmente de acuerdo" o muy positivo") su opinión o grado de conformidad con la misma.

La recogida de información se realizó durante la última semana del período de clases del curso 2010-2011. Dos miembros del equipo investigador debidamente formados administraron el cuestionario, de carácter anónimo y voluntario, durante las horas lectivas de los estudiantes y con la autorización previa por parte del profesor 
responsable. Los encuestadores presentaron a los participantes el cuestionario, dando las instrucciones pertinentes para su cumplimentación y contestando a cualquier duda que pudiese surgir.

\section{Diseño y validación del cuestionario}

Se diseñó un cuestionario inicial de 30 ítems que fue sometido a la consideración de cuatro expertos reunidos en tres ocasiones con estructura de trabajo de grupo de discusión. El cuestionario consensuado finalmente reunía 28 ítems agrupados en cuatro ámbitos: aspectos generales de los estudios; organización académica de la universidad y la facultad: enseñanza y metodología docente; y opinión sobre el Plan Bolonia.

Se procedió a analizar la homogeneidad de los ítems calculando la correlación de cada uno de ellos con la puntuación total de la escala. El cambio en la consistencia interna fue evaluado comprobando la modificación del coeficiente alfa de Cronbach al eliminar cada uno de los ítems.

En un segundo análisis se procedió a comprobar el poder discriminativo de cada ítem mediante pruebas t-Student para muestras independientes, comparando las puntuaciones de los sujetos que superaban el percentil 75 de la puntuación total de la escala con aquellos que no superaban el percentil 25. El objetivo de este análisis fue detectar aquellos ítems que no contribuían a la dimensión evaluada por la escala.

Los criterios seguidos para la no eliminación de los ítems fueron:

1. Que la correlación del ítem con la escala fuera superior a 0.30 excluido el propio ítem.

2. Que el coeficiente alfa de Cronbach de la escala no aumentase si se eliminaba ese ítem.

3. Que los ítems tuviesen suficiente poder discriminativo, es decir: que se obtuviese una diferencia significativa entre las puntuaciones medias del ítem al comparar los grupos extremos.

De acuerdo al segundo criterio, fueron eliminados 2 de los 28 ítems iniciales, resultando un cuestionario final de 26 ítems.

El análisis factorial exploratorio (método de máxima verosimilitud, rotación varimax) permitió extraer dos factores que explicaron conjuntamente un $47.03 \%$ de la varianza (Tabla 2).

Tabla 2. Autovalores y porcentajes de varianza explicada de los factores extraídos

Factor $1 \quad$ Factor 2

\begin{tabular}{lll}
\hline Autovalores & 9.02 & 3.21 \\
\hline$\%$ Varianza & 34.71 & 12.33 \\
\hline$\%$ Varianza acumulado & 34.71 & 47.03
\end{tabular}


Las medidas de calidad inicial del análisis resultaron ser muy satisfactorias, con un índice de adecuación muestral de $\mathrm{KMO}=0.932$ y una prueba de esfericidad de Bartlett significativa (chi-cuadrado $=6398.80, \mathrm{gl}=325, \mathrm{p}>0.001$ ). El factor primero recogió 21 de los 26 ítems resultantes, todos ellos pertenecientes a tres de los ámbitos preestablecidos: aspectos generales, organización académica y enseñanza; mostrando un coeficiente alpha de 0.91 . El segundo factor estuvo formado por los 5 restantes, coincidentes con los que conformaban el ámbito correspondiente al Plan Bolonia; mostrando un coeficiente alpha de 0.94 .

\section{Resultados y Discusión}

Se realizó una comparación de medias de los resultados globales de cada factor (prueba de MannWhitney), encontrando que los estudiantes de grado mostraron puntuaciones significativamente más elevadas que los estudiantes de licenciatura en ambos: factor $1(\mathrm{Z}=-6.83 ; \mathrm{p}<0.001)$ y factor $2(\mathrm{Z}=-2.45 ; \mathrm{p}>0.05)$.

Esta relación se mantenía al comparar las medias por ámbitos: aspectos generales $(\mathrm{Z}=-8.57 ; \mathrm{p}<0.001)$, organización académica $(\mathrm{Z}=-4.54 ; \mathrm{p}<0.001)$, enseñanza $(\mathrm{Z}=-$ 4.28; $\mathrm{p}<0.001)$, y Plan Bolonia $(\mathrm{Z}=-2.45 ; \mathrm{p}>0.05)$ (Tabla 3 ).

Tabla 3. Diferencias de medias encontradas por factores y ámbitos según titulación de los estudiantes

\begin{tabular}{lccc}
\hline & Grado & Licenciatura & p. \\
& M (DT) & M (DT) & $<\mathbf{3 . 0 1}$ \\
\hline Factor 1 & $\mathbf{3 . 5 1}(\mathbf{. 5 8})$ & $\mathbf{3 . 1 3}(\mathbf{. 5 9 )}$ & $<0.01$ \\
Aspectos generales & $3.88(.65)$ & $3.32(.71)$ & $<0.01$ \\
Organización académica & $3.40(.68)$ & $3.10(.69)$ & $<0.01$ \\
Enseñanza & $3.24(.64)$ & $2.97(.60)$ & $<\mathbf{0 . 0 5}$ \\
Factor 2 & $\mathbf{2 . 6 9 ( 1 . 1 2 )}$ & $\mathbf{2 . 5 3 ( 1 . 3 0 )}$ & $<0.05$ \\
$\quad$ Plan Bolonia & $2.69(1.12)$ & $2.53(1.30)$ & \\
\hline
\end{tabular}

Dado que los factores resultantes agrupaban perfectamente los ítems de los ámbitos establecidos previamente se decidió, de acuerdo con los expertos, presentar los resultados por ámbitos ya que, metodológicamente, suponía una mayor sencillez y claridad en la exposición y una mejor interpretación en su lectura.

\section{Aspectos Generales}

Existen diferencias significativas en todos los ítems de este ámbito entre estudiantes de grado y licenciatura, siendo en todos los casos más elevada la valoración que hacen los primeros.

La Tabla 4 muestra los porcentajes obtenidos para cada ítem, así como la media y la desviación típica con que cada grupo de estudiantes (grado o licenciatura), lo valora. Además de las diferencias de medias entre ambos colectivos, se observa que los estudiantes de licenciatura muestran mayores porcentajes en las respuestas más bajas 
(entre 1 y 3), mientras que los de grado lo hacen en las puntuaciones más elevadas (entre 4 y 5). Cabe destacar los resultados obtenidos en el ítem Grado de satisfacción con los estudios que estoy cursando, en el que el porcentaje de estudiantes de grado que lo valoran de forma positiva o muy positiva (71\%) supera en más del doble al porcentaje de estudiantes de licenciatura (34\%).

Tabla 4. Resultados de la opinión del alumnado respecto a aspectos generales de sus estudios universitarios

\begin{tabular}{|c|c|c|c|c|c|c|}
\hline & $\mathrm{M}(\mathrm{DT})$ & 1 & 2 & 3 & 4 & 5 \\
\hline $\begin{array}{l}\text { La carrera que estoy estudiando es a la que } \\
\text { aspiraba cuando realicé la preinscripción } \\
\text { Grado } \\
\text { Licenciatura }\end{array}$ & $\begin{array}{l}4.40(0.91) \\
3.79(1.09)\end{array}$ & $\begin{array}{l}1 \% \\
3 \%\end{array}$ & $\begin{array}{l}4 \% \\
10 \%\end{array}$ & $\begin{array}{l}12 \% \\
25 \%\end{array}$ & $\begin{array}{l}21 \% \\
30 \%\end{array}$ & $\begin{array}{l}62 \% \\
32 \%\end{array}$ \\
\hline $\begin{array}{l}\text { Grado de satisfacción con los estudios que } \\
\text { estoy cursando } \\
\text { Grado } \\
\text { Licenciatura }\end{array}$ & $\begin{array}{l}3.88(0.93) \\
3.19(0.93)\end{array}$ & $\begin{array}{l}1 \% \\
5 \%\end{array}$ & $\begin{array}{l}8 \% \\
14 \%\end{array}$ & $\begin{array}{l}20 \% \\
46 \%\end{array}$ & $\begin{array}{l}44 \% \\
27 \%\end{array}$ & $\begin{array}{l}27 \% \\
7 \%\end{array}$ \\
\hline $\begin{array}{l}\text { En líneas generales, la valoración que hago } \\
\text { de la universidad } \\
\text { Grado } \\
\text { Licenciatura }\end{array}$ & $\begin{array}{l}3.67(0.79) \\
3.24(0.97)\end{array}$ & $\begin{array}{l}1 \% \\
5 \%\end{array}$ & $\begin{array}{l}7 \% \\
15 \%\end{array}$ & $\begin{array}{l}27 \% \\
38 \%\end{array}$ & $\begin{array}{l}54 \% \\
37 \%\end{array}$ & $\begin{array}{l}11 \% \\
4 \%\end{array}$ \\
\hline $\begin{array}{l}\text { En líneas generales, la valoración que hago } \\
\text { de la facultad } \\
\text { Grado } \\
\text { Licenciatura }\end{array}$ & $\begin{array}{l}3.82(0.82) \\
3.18(0.93)\end{array}$ & $\begin{array}{l}0 \% \\
5 \%\end{array}$ & $\begin{array}{l}6 \% \\
15 \%\end{array}$ & $\begin{array}{l}24 \% \\
41 \%\end{array}$ & $\begin{array}{l}51 \% \\
33 \%\end{array}$ & $\begin{array}{l}18 \% \\
5 \%\end{array}$ \\
\hline $\begin{array}{l}\text { Opinión sobre el nivel de formación que } \\
\text { recibo en la carrera } \\
\text { Grado } \\
\text { Licenciatura }\end{array}$ & $\begin{array}{l}3.65(0.90) \\
3.18(0.97)\end{array}$ & $\begin{array}{l}2 \% \\
5 \%\end{array}$ & $\begin{array}{l}6 \% \\
18 \%\end{array}$ & $\begin{array}{l}33 \% \\
39 \%\end{array}$ & $\begin{array}{l}42 \% \\
32 \%\end{array}$ & $\begin{array}{l}16 \% \\
5 \%\end{array}$ \\
\hline
\end{tabular}

Atendiendo al nivel de formación recibido en la carrera, el 58\% de los estudiantes de grado lo valora positivamente, frente a un $37 \%$ de los de licenciatura. Como indican las propuestas de renovación de las metodologías en la universidad (Consejo de Coordinación Universitaria, 2006) con el nuevo plan no se cuestiona la necesidad y la utilidad educativa de la formación práctica, y es éste uno de los puntos de divergencia con respecto al sistema anterior. En los nuevos estudios se tiende a reforzar este tipo de formación, bien a través de prácticas dentro de las asignaturas, como a través de prácticas externas que preparen para la habilitación profesional.

\section{Organización Académica}

En este ámbito también se encuentran diferencias significativas en todos los ítems en el mismo sentido que el ámbito anterior: en todos los casos las puntuaciones de los alumnos de grado son más elevadas que las de alumnos de licenciatura. La Tabla 5 muestra los resultados globales en términos de medias, desviaciones típicas y porcentajes de cada valoración obtenidos para cada ítem. 
Tabla 5. Resultados de la opinión del alumnado respecto a la organización académica de su universidad (\%)

\begin{tabular}{|c|c|c|c|c|c|c|}
\hline & $\mathrm{M}$ (DT) & 1 & 2 & 3 & 4 & 5 \\
\hline $\begin{array}{l}\text { Tengo información suficiente sobre los } \\
\text { aspectos académicos de los estudios que } \\
\text { estoy cursando } \\
\text { Grado } \\
\text { Licenciatura }\end{array}$ & $\begin{array}{l}.44(0.93) \\
3.16(0.95) \\
\end{array}$ & $\begin{array}{c}3.2 \% \\
5 \% \\
\end{array}$ & $\begin{array}{c}11 \% \\
17.9 \% \\
\end{array}$ & $\begin{array}{l}35.1 \% \\
38.3 \% \\
\end{array}$ & $\begin{array}{l}40.4 \% \\
33.8 \% \\
\end{array}$ & $\begin{array}{c}10.3 \% \\
5 \% \\
\end{array}$ \\
\hline $\begin{array}{l}\text { Cuando he tenido dudas me las han } \\
\text { resuelto adecuadamente } \\
\text { Grado } \\
\text { Licenciatura }\end{array}$ & $\begin{array}{l}3.38(0.98) \\
3.18(0.97)\end{array}$ & $\begin{array}{c}5.7 \% \\
3 \%\end{array}$ & $\begin{array}{l}10.6 \% \\
21.4 \%\end{array}$ & $\begin{array}{c}33 \% \\
38.8 \% \\
\end{array}$ & $\begin{array}{l}41.8 \% \\
28.9 \%\end{array}$ & $\begin{array}{l}8.9 \% \\
7.5 \%\end{array}$ \\
\hline $\begin{array}{l}\text { El número de horas que invierto en cada } \\
\text { asignatura corresponde con los créditos } \\
\text { asignados } \\
\text { Grado } \\
\text { Licenciatura }\end{array}$ & $\begin{array}{l}3.13(1.08) \\
2.85(1.02)\end{array}$ & $\begin{array}{c}8.2 \% \\
11.4 \% \\
\end{array}$ & $\begin{array}{l}18.8 \% \\
22.4 \% \\
\end{array}$ & $\begin{array}{l}33.7 \% \\
40.3 \% \\
\end{array}$ & $\begin{array}{l}31.2 \% \\
21.9 \% \\
\end{array}$ & $\begin{array}{c}7.4 \% \\
4 \% \\
\end{array}$ \\
\hline $\begin{array}{l}\text { El horario de clases es adecuado y facilita } \\
\text { el aprendizaje } \\
\text { Grado } \\
\text { Licenciatura }\end{array}$ & $\begin{array}{l}3.42(1.11) \\
2.98(1.08)\end{array}$ & $\begin{array}{c}7.4 \% \\
10.4 \%\end{array}$ & $\begin{array}{l}11.3 \% \\
21.4 \%\end{array}$ & $\begin{array}{l}28.7 \% \\
35.3 \%\end{array}$ & $\begin{array}{l}37.2 \% \\
25.9 \%\end{array}$ & $\begin{array}{c}14.9 \% \\
7 \%\end{array}$ \\
\hline $\begin{array}{l}\text { El calendario de exámenes es adecuado } \\
\text { Grado } \\
\text { Licenciatura }\end{array}$ & $\begin{array}{l}3.40(1.18) \\
3.02(1.21)\end{array}$ & $\begin{array}{c}8.5 \% \\
13.9 \% \\
\end{array}$ & $\begin{array}{l}13.5 \% \\
18.9 \% \\
\end{array}$ & $\begin{array}{l}26.2 \% \\
29.4 \% \\
\end{array}$ & $\begin{array}{c}33 \% \\
26.4 \% \\
\end{array}$ & $\begin{array}{l}18.8 \% \\
11.4 \% \\
\end{array}$ \\
\hline $\begin{array}{l}\text { Las instalaciones y el material para la } \\
\text { impartición de las clases es adecuado } \\
\text { Grado } \\
\text { Licenciatura }\end{array}$ & $\begin{array}{l}3.67(0.96) \\
3.43(1.24)\end{array}$ & $\begin{array}{l}3.2 \% \\
9.5 \% \\
\end{array}$ & $\begin{array}{c}9.2 \% \\
12.4 \% \\
\end{array}$ & $\begin{array}{l}24.5 \% \\
26.9 \% \\
\end{array}$ & $\begin{array}{l}44.3 \% \\
28.4 \% \\
\end{array}$ & $\begin{array}{l}18.4 \% \\
22.9 \% \\
\end{array}$ \\
\hline
\end{tabular}

Atendiendo a las cuestiones planteadas, se diferencian dos agrupaciones: una constituida por los dos primeros ítems del ámbito y relacionada con la opinión de los estudiantes sobre la información recibida; y una segunda que agrupa los cuatro últimos ítems del ámbito y hace referencia a la gestión y organización académica percibidas.

En lo concerniente a la información recibida por el alumnado, desde la propia facultad se han llevado a cabo diversas acciones. El programa de acogida para estudiantes de nuevo ingreso (curso 0 ) ha sido reformulado para responder a las nuevas demandas realizándose una presentación de la facultad al nuevo alumnado así como charlas informativas sobre las reformas que acarrea la puesta en marcha del Plan Bolonia tanto para estos estudiantes, como para aquellos del plan anterior que se encuentran cursando la licenciatura.

Los resultados sugieren que las iniciativas llevadas a cabo para informar a alumnos de nuevo ingreso han sido adecuadas. Aunque era de esperar que alumnos pertenecientes al plan anterior hubiesen valorado más positivamente la información recibida sobre los aspectos académicos de sus estudios, ya que han estado durante más 
años en la facultad, han sido los estudiantes de grado quienes alcanzaron mayores puntuaciones en este ítem. A pesar de estas diferencias, si comparamos las puntuaciones con las alcanzadas en el ámbito anterior, éstas son más bajas. Como se aprecia en la Tabla 3, el porcentaje de estudiantes de grado que valora positivamente el nivel de información que posee sobre los aspectos académicos de sus estudios ronda el $50 \%$; y en el caso de estudiantes de licenciatura ni siquiera alcanza el $40 \%$. En un estudio de similares características, (Castaño, et al., 2007) encontraron que un alto porcentaje del alumnado de grado analizado (23\%) considera que no ha recibido información sobre el proceso EEES, exponiendo como posible justificación las complicaciones encontradas en el inicio del curso académico y la tardía incorporación de un elevado número de estudiantes, lo que impidió que estos acudieran a las reuniones informativas establecidas. En el caso del presente trabajo, y pese a haber obtenido mejores resultados, es posible que el cambio al que se enfrenta el alumnado sea tan grande, y genere tal incertidumbre, que se debía haber intensificado la información procurada sobre los cambios que acarrea el Plan Bolonia.

$\mathrm{Si}$ profundizamos en el análisis, encontramos que la diferencia entre ambos colectivos en el ítem que nos ocupa (información recibida) $\left(\mathrm{M}_{\text {Grado }}=3.44, \mathrm{DT}_{\text {Grado }}=\right.$ $\left.0.93 ; \mathrm{M}_{\text {Licenciatura }}=3.16, \mathrm{DT}_{\text {Licenciatura }}=0.95\right)$ sugiere que la reformulación del programa de acogida para nuevos estudiantes, con la inclusión de charlas específicas sobre la adaptación al EEES, fue más efectiva que las reuniones convocadas para los estudiantes de ambos planes como medida informativa, lo cual nos induce a pensar en la efectividad del programa y la posibilidad de utilizarlo en un futuro para la divulgación de otras informaciones relevantes que transmitir al alumnado.

En el análisis del segundo grupo de ítems, referido a la gestión y organización académica, apreciamos notables diferencias en la percepción que tiene el alumnado sobre la adecuación del horario para facilitar el aprendizaje; mientras más del $50 \%$ de los estudiantes de grado valoran positivamente el horario de clases, los de licenciatura no alcanzan el $34 \%$. Frente a un horario de segmentos de duración variable que finaliza a media tarde en licenciatura, el alumnado de grado está más satisfecho con un modelo de horario académico que concentra las clases por la mañana, dejando las tardes libres, y en el que todos los bloques son de 2 horas (excepto las asignaturas básicas de deporte impartidas en primero y segundo de grado, de 1 hora y media). Las características del horario del nuevo plan son más similares a las del horario de bachillerato, de donde proceden la mayoría de estudiantes que acceden a estos estudios. El hecho de continuar con una rutina horaria llevada a cabo durante años, que llegada a esta edad le permite compatibilizar sus estudios de grado con cualquier otra actividad a realizar durante las tardes, puede ser un motivo clave para entender el porqué de esta mejor valoración sobre los horarios nuevos que sobre los antiguos.

Son destacables también las discrepancias encontradas en la opinión de alumnado sobre el calendario de exámenes. Los estudiantes de grado se muestran más satisfechos $(\mathrm{M}=3.40 ; \mathrm{DT}=1.18)$ que los de licenciatura $(\mathrm{M}=3.02$; $\mathrm{DT}=1.21)$. En el EEES, las asignaturas pasan a ser semestrales, desaparece la convocatoria anual y la matrícula de asignaturas se realiza en dos momentos, septiembre y febrero, teniendo lugar las dos convocatorias de cada asignatura antes de cerrar la matrícula del siguiente período. 
Parece que el alumnado entiende que la existencia de objetivos más cercanos (evaluaciones semestrales frente a anuales) es más adecuada para un proceso favorable de aprendizaje.

\section{Enseñanza}

En este ámbito se encuentran diferencias significativas en todos los ítems, presentando también valoraciones más elevadas los alumnos de grado que de licenciatura, excepto en los ítems La relación profesor-alumno y Las tutorías me han facilitado la comprensión y asimilación de los contenidos, en los que no se aprecian diferencias en ningún sentido. Como hemos hecho en los ámbitos anteriores, en la Tabla 6 se muestran los resultados del ámbito Enseñanza en términos de porcentajes obtenidos en cada puntuación según la titulación a la que perteneciesen los estudiantes.

Tabla 6. Resultados de la opinión del alumnado respecto al proceso de enseñanza-aprendizaje

\begin{tabular}{|c|c|c|c|c|c|c|}
\hline & $\mathrm{M}(\mathrm{DT})$ & 1 & 2 & 3 & 4 & 5 \\
\hline \multicolumn{7}{|c|}{$\begin{array}{l}\text { La calidad de la enseñanza que } \\
\text { recibo }\end{array}$} \\
\hline Grado & $3.63(0.83)$ & $0.4 \%$ & $9.2 \%$ & $30.1 \%$ & $47.5 \%$ & $12.8 \%$ \\
\hline Licenciatura & $3.18(0.82)$ & $3 \%$ & $14.9 \%$ & $44.8 \%$ & $35.3 \%$ & $2 \%$ \\
\hline \multicolumn{7}{|c|}{$\begin{array}{l}\text { La metodología de enseñanza } \\
\text { empleada en clase }\end{array}$} \\
\hline Grado & $3.38(0.87)$ & $1.8 \%$ & $11.3 \%$ & $40.1 \%$ & $40.4 \%$ & $6.4 \%$ \\
\hline Licenciatura & $2.96(0.85)$ & $6.5 \%$ & $16.9 \%$ & $52.7 \%$ & $21.9 \%$ & $2 \%$ \\
\hline \multicolumn{7}{|c|}{ La relación profesor-alumno } \\
\hline Grado & $3.46(0.94)$ & $2.5 \%$ & $12.4 \%$ & $33.3 \%$ & $40.1 \%$ & $11.7 \%$ \\
\hline Licenciatura & $3.31(0.92)$ & $2.5 \%$ & $15.9 \%$ & $37.3 \%$ & $36.3 \%$ & $8 \%$ \\
\hline \multicolumn{7}{|l|}{$\begin{array}{l}\text { El sistema de evaluación } \\
\text { utilizado }\end{array}$} \\
\hline Grado & $3.35(0.96)$ & $5.3 \%$ & $9.6 \%$ & $39 \%$ & $37.2 \%$ & $8.9 \%$ \\
\hline Licenciatura & $3.12(0.85)$ & $3 \%$ & $18.4 \%$ & $44.8 \%$ & $30.8 \%$ & $3 \%$ \\
\hline \multicolumn{7}{|c|}{$\begin{array}{l}\text { El grado de utilización de las } \\
\text { TIC }\end{array}$} \\
\hline Grado & $3.45(1.11)$ & $4.3 \%$ & $15.2 \%$ & $30.9 \%$ & $30.5 \%$ & $18.4 \%$ \\
\hline Licenciatura & $2.83(1.02)$ & $10 \%$ & $26.4 \%$ & $37.8 \%$ & $21.4 \%$ & $4.5 \%$ \\
\hline \multicolumn{7}{|c|}{$\begin{array}{l}\text { Las tutorías me han facilitado la } \\
\text { comprensión y asimilación de } \\
\text { los contenidos }\end{array}$} \\
\hline Grado & $2.74(1.44)$ & $26.6 \%$ & $18.1 \%$ & $26.2 \%$ & $18.4 \%$ & $5.3 \%$ \\
\hline Licenciatura & $2.88(1.26)$ & $18.9 \%$ & $16.4 \%$ & $32.3 \%$ & $24.4 \%$ & $6 \%$ \\
\hline \multicolumn{7}{|c|}{$\begin{array}{l}\text { La cantidad de prácticas es } \\
\text { adecuada }\end{array}$} \\
\hline Grado & $3.32(1.07)$ & $5.3 \%$ & $16 \%$ & $33.7 \%$ & $32.6 \%$ & $11.7 \%$ \\
\hline Licenciatura & $3.06(1.05)$ & $7.5 \%$ & $21.4 \%$ & $36.8 \%$ & $25.9 \%$ & $8.5 \%$ \\
\hline
\end{tabular}


La cantidad de trabajo personal necesario para superar las asignaturas es adecuado
Grado
Licenciatura

$3.32(1.00)$

$3.17(0.84)$
He recibido evaluaciones

periódicas sobre el trabajo

personal realizado

Grado
Licenciatura

Lo aprendido es válido para mi futuro profesional

Grado
Licenciatura

$3.13(1.06)$

$2.69(1.05)$
$14.9 \%$

$9 \%$

\begin{tabular}{ll}
$3.9 \%$ & $14.9 \%$ \\
$5 \%$ & $9 \%$ \\
\hline
\end{tabular}

\begin{abstract}
$18.1 \%$
\end{abstract}
$7.4 \%$
$12.9 \%$

$30.3 \%$

\begin{tabular}{l}
$37.2 \%$ \\
$36.8 \%$ \\
\hline
\end{tabular}

$38.3 \%$

$54.2 \%$

$9.6 \%$

$4 \%$

$\begin{array}{ll}32.3 \% & \\ 27.9 \% & 4 \%\end{array}$

Los resultados del tercer ámbito continúan en la línea de los anteriores y, de este modo, los estudiantes de grado presentan puntuaciones más elevadas que los de licenciatura en lo referente a la calidad de la enseñanza. Ésta es valorada como positiva o muy positiva por más del $60 \%$ de estudiantes de grado, valoración que no alcanza el $40 \%$ entre estudiantes de licenciatura. Estos resultados podrían estar relacionados con los obtenidos en la valoración del alumnado acerca de la metodología empleada en clase. Este aspecto es valorado como positivo o muy positivo por un $47 \%$ de los estudiantes de grado, mientras que no llega al $24 \%$ entre los de licenciatura. Resulta lógico pensar que el cambio realizado en la metodología en el proceso de adaptación al EEES pueda estar relacionado, y ser un predictor de la calidad percibida en la enseñanza en tanto que la metodología de enseñanza es uno de los aspectos clave de un proceso educativo que más fácilmente perciben los estudiantes.

Sin embargo, la relación que encontramos entre la calidad de enseñanza percibida y la metodología de enseñanza no está en línea con las opiniones del alumnado acerca de la relación profesor-alumno, aspecto sobre el que tanto estudiantes de grado como de licenciatura muestran un mayor acuerdo $\left(\mathrm{M}_{\mathrm{Grado}}=3.46, \mathrm{DT}_{\mathrm{Grado}}=0.94 ; \mathrm{M}_{\text {Licenciatura }}=\right.$ 3.31, $\mathrm{DT}_{\text {Licenciatura }}=0.92$ ). La mayor parte del profesorado imparte o ha impartido docencia en ambos planes, lo que puede explicar que no se encuentren diferencias significativas en la relación profesor-alumno percibida por estudiantes de grado o por estudiantes de licenciatura. Este aspecto parece estar al margen de la metodología empleada en clase, lo que nos indica que la formación continua del profesorado universitario es fundamental para actualizar los métodos de enseñanza. Las características individuales de los profesores parecen no influir en tanta medida sobre la percepción de la calidad de la enseñanza como lo hacen las metodologías empleadas en clase.

También cabe destacar la valoración positiva de estudiantes de grado sobre la adecuación de la cantidad de prácticas realizadas, un $45 \%$ se muestra satisfecho o muy satisfecho; o la validez de lo aprendido para enfrentarse a su futuro profesional, ítem con el que el porcentaje de satisfechos o muy satisfechos asciende a un 54\%. Esta información no hace sino reforzar lo encontrado en el análisis del primer ámbito 
analizado de que la formación, basada ahora en actividades más prácticas que teóricas, es más adecuada para enfrentarse al futuro laboral que espera a los estudiantes una vez finalizados sus estudios. Estos resultados coinciden con lo encontrado en otros estudios como el de Montaño (2008) en el que más de un 70\% de los estudiantes de Grado encuestados se muestran muy satisfechos con el aprendizaje encontrado.

En este tercer ámbito aparece un elemento que, siendo clave en cualquier proceso educativo, adquiere una relevancia especial en el Plan Bolonia por ser uno de los pilares del modelo: la personalización de la enseñanza a través de las tutorías. Es precisamente en lo concerniente a ellas donde existe un alto porcentaje de respuestas negativas, siendo más elevado este porcentaje entre estudiantes de grado. Si nos remitimos de nuevo a las conclusiones de las propuestas para la renovación de las metodologías educativas en la universidad (Consejo de Coordinación Universitaria, 2006), encontramos que son las universidades privadas las que están apostando más decididamente por la expansión de estas tutorías individualizadas. Posiblemente el elevado volumen de alumnado que se encuentra en la universidad pública, así como el hecho de que la adaptación al EEES se encuentra aún en una fase de recién estrenada implantación, hacen que el papel de las tutorías, de momento, no resulte tan clave como la renovación sugerida por el Plan Bolonia podría hacernos pensar. Este aspecto requiere, dada su relevancia, de ulteriores estudios que permitan una profundización en los factores que lo caracterizan.

\section{Plan Bolonia}

En este último ámbito se aprecian diferencias significativas en las puntuaciones otorgadas por estudiantes de grado y licenciatura en todos los ítems excepto en el de Bolonia dispone de más recursos para la enseñanza. En el resto, las puntuaciones de estudiantes de grado son más elevadas que las de estudiantes de licenciatura. En la Tabla 7 se muestran los resultados de la opinión de alumnado con respecto al Plan Bolonia. Son destacables los elevados porcentajes de respuestas que muestran un alto desacuerdo en que el Plan Bolonia conlleve mejora. En el ítem Bolonia mejora la universidad, la respuesta 1 ("muy en desacuerdo") fue elegida por el $42 \%$ de los estudiantes de licenciatura. Por el contrario, en ninguno de los ítems se alcanza el 10\% de respuestas 5 ("muy de acuerdo"). El desconocimiento que los estudiantes muestran sobre Bolonia podría estar relacionado con la tardía adaptación de las universidades españolas al EEES, aspecto comentado anteriormente.

Tabla 7. Resultados de la opinión del alumnado respecto al Plan Bolonia

\begin{tabular}{cclllll}
\hline Bolonia... & M (DT) & 1 & 2 & 3 & 4 & 5 \\
\hline $\begin{array}{l}\text { mejora la organización } \\
\text { académica de la facultad }\end{array}$ & & & & & & \\
$\quad$ Grado & $2.63(1.16)$ & $20.9 \%$ & $24.1 \%$ & $31.2 \%$ & $18.8 \%$ & $3.2 \%$ \\
$\quad$ Licenciatura & $2.40(1.29)$ & $32.3 \%$ & $23.4 \%$ & $24.4 \%$ & $12.4 \%$ & $2.5 \%$ \\
\hline $\begin{array}{l}\text { mejora la calidad de la } \\
\text { enseñanza }\end{array}$ & $2.55(1.16)$ & $23.8 \%$ & $23.8 \%$ & $30.1 \%$ & $18.1 \%$ & $2.8 \%$ \\
$\quad$ Grado & $2.37(1.31)$ & $34.3 \%$ & $22.9 \%$ & $23.9 \%$ & $10.9 \%$ & $2 \%$ \\
$\quad$ Licenciatura & & & & & & \\
\hline
\end{tabular}




\begin{tabular}{lllllll}
\hline $\begin{array}{l}\text { dispone de más recursos para la } \\
\text { enseñanza }\end{array}$ & & & & & & \\
$\quad$ Grado & $2.65(1.16)$ & $22 \%$ & $20.9 \%$ & $31.9 \%$ & $20.9 \%$ & $2.8 \%$ \\
$\quad$ Licenciatura & $2.62(1.33)$ & $26.9 \%$ & $20.4 \%$ & $27.4 \%$ & $15.9 \%$ & $2.5 \%$ \\
\hline $\begin{array}{l}\text { forma profesionales mejor } \\
\text { preparados para el mercado }\end{array}$ & & & & & & \\
laboral & $2.67(1.22)$ & $21.6 \%$ & $22 \%$ & $31.9 \%$ & $16.3 \%$ & $6 \%$ \\
$\quad$ Grado & $2.39(1.44)$ & $38.3 \%$ & $20.9 \%$ & $18.4 \%$ & $10.9 \%$ & $4 \%$ \\
$\quad$ Licenciatura & & & & & & \\
\hline $\begin{array}{l}\text { mejora la universidad } \\
\text { Grado }\end{array}$ & $2.51(1.20)$ & $26.2 \%$ & $22.3 \%$ & $31.9 \%$ & $13.1 \%$ & $4.3 \%$ \\
$\quad$ Licenciatura & $2.20(1.35)$ & $42.3 \%$ & $22.9 \%$ & $18.9 \%$ & $6.5 \%$ & $2 \%$ \\
\hline
\end{tabular}

Pese a las claras diferencias encontradas entre ambos colectivos en los ámbitos anteriores que muestran una constatación de cambio en el modelo, parece que el discurso de los estudiantes al ser preguntados directamente sobre Bolonia es de desconfianza o poca convicción. Su posicionamiento teórico no coincide con su visión de los aspectos específicos de aplicación del nuevo modelo (p. ej. metodología, horario), lo que nos sugiere que, pese a mantener un discurso ligeramente reactivo al cambio, sin embargo las evidencias prácticas del mismo son valoradas positivamente.

Como último elemento de nuestro análisis, se realizó una comparación de medias ámbito por ámbito para analizar posibles diferencias según el género del alumnado, no encontrándose diferencias significativas (Aspectos Generales $\mathrm{Z}=-1.07, \mathrm{p}>0.5$; Organización Académica $\mathrm{Z}=-1.01, \mathrm{p}>0.5$; Enseñanza $\mathrm{Z}=-0.21, \mathrm{p}>0.5$ y Bolonia $\mathrm{Z}=-$ $0.45, \mathrm{p}>0.5$ ) entre hombres y mujeres.

\section{Conclusiones}

En pocos años, la universidad ha puesto en marcha procesos de cambio organizativo y metodológico. En lo referente a esta renovación es importante hacer partícipes a los estudiantes, y no exclusivamente a los docentes, en la toma de decisiones y formulación de opiniones en lo que respecta a la transformación que está teniendo lugar. Por ello decidimos analizar qué cambios han tenido lugar en la percepción de los estudiantes acerca de diferentes aspectos relacionados con la vida académica que han sufrido modificaciones con el Plan Bolonia.

A la vista de los resultados obtenidos en el presente estudio, resulta interesante y contradictorio lo que se observa acerca de la percepción del alumnado sobre los cambios que acarrea Bolonia. Atendiendo a las valoraciones generales por ámbitos encontramos cómo, al hablar explícitamente del Plan Bolonia, se obtienen las puntuaciones más bajas tanto por parte de los estudiantes de grado como de licenciatura, lo que nos llevaría a interpretar que el alumnado no valora como positivos los cambios introducidos por el nuevo modelo. Sin embargo, profundizando un poco más en lo relativo a dichos cambios, hemos encontrado diferencias significativas en el resto de ámbitos analizados según los estudiantes perteneciesen al plan antiguo o al nuevo. Como hemos visto, en todos los casos los estudiantes de grado 
se han mostrado más satisfechos que los estudiantes de licenciatura, lo que nos lleva a pensar que la implantación del EEES ha supuesto una mejora tanto en cuestiones institucionales como de enseñanza. Es posible que los estudiantes de licenciatura, influenciados por la avalancha de protestas que han tenido lugar con la puesta en marcha del plan nuevo, no confiasen en que la incorporación al EEES supusiese las mejoras a las que el cuestionario hace mención; mientras que quizás, los estudiantes de grado, no sean conscientes del papel del Plan Bolonia como agente de cambio en los aspectos evaluados al no haber vivenciado la realidad anterior.

La mayor satisfacción del alumnado con cuestiones institucionales que con las de enseñanza es explicable si consideramos la tardía incorporación de España al proceso implantado por Bolonia que hemos comentado anteriormente. Este retraso, en comparación con otros países participantes, hace que nuestras universidades estén aún dedicando sus esfuerzos a que los alumnos de grado tengan una completa información sobre la organización académica que el nuevo plan conlleva; dejando en un segundo plano lo concerniente a la metodología de enseñanza. Este proceso de actualización e innovación educativa, en lo relativo a los métodos didácticos utilizados por el profesor, requiere más tiempo y esfuerzo, por lo que no son de extrañar los resultados obtenidos en este trabajo. Es precisamente en esta línea de trabajo, de actualización y adaptación de la metodología de enseñanza a lo requerido por las características del nuevo EEES, donde pensamos que habrá que centrarse en los próximos años para continuar con una correcta implantación del nuevo plan en nuestro país.

Con el fin de complementar la información recabada en el presente trabajo, nos proponemos continuar esta línea de investigación mediante la realización de entrevistas tanto a profesores como a estudiantes que, habiendo comenzado sus estudios de licenciatura, se han cambiado posteriormente al grado. Desde esta triple perspectiva, dispondremos de material suficiente que nos permita comprender el modo en que se está llevando a cabo la implantación de los nuevos estudios de grado en la FCCAFD, para, a partir de este conocimiento, adoptar las medidas más adecuadas para la mejora de dicho proceso.

Sería interesante comprobar en un estudio posterior si el hecho de que los estudiantes de grado encuestados sean precisamente estudiantes de nuevo ingreso en la facultad pueda influir en los resultados obtenidos ya que normalmente la motivación inicial con que los estudiantes comienzan su andadura universitaria va desciendo a medida que avanzan de curso.

\section{Referencias bibliográficas}

ANECA. (2005). Libro blanco. Título de grado en ciencias de la actividad física y del deporte. Disponible en http://www.aneca.es/var/media/150296/libroblanco_deporte_def.pdf

CASTAÑO, E., BENITO, Á., PORTElA, A. Y RODRÍGUEZ, R. M. (2007). Repercusiones en los alumnos de primer curso de la implantación del Espacio Europeo. Revista complutense de educación, 18(1), 199-216. 
COMUNICADO DE BERGEN. (2005). El Espacio Europeo de Educación Superior. Alcanzando las metas. Comunicado de la Conferencia de Ministros Europeos responsables de Educación Superior. Bergen, 19-20 de mayo del 2005. Disponible en http://www.crue.org/export/sites/Crue/procbolonia/documentos/antecedentes/ 6._Comunicado_de_Bergen.pdf

COMUNICADO DE BERLÍN. (2003). Comunicado de la Conferencia de Ministros responsables de la Educación Superior. Berlín, 19 de septiembre del 2003. Disponible en http: //www.eees.es/pdf/Berlin_ES.pdf

CONSEJO DE COORDINACIÓN UNIVERSITARIA. (2006). Propuesta para la renovación de las metodologías educativas en la universidad. Madrid: Ministerio de Educación y Ciencia. Disponible en http://www.upcomillas.es/eees/ Documentos/PROPUESTA_RENOVACION. pdf

DECLARACIÓN DE BOLONIA. (1999). Declaración de Bolonia. El Espacio Europeo de la Enseñanza Superior. Declaración conjunta de los Ministros europeos de Educación, Bolonia 19 de junio de 1999. Disponible en http://www.educacion.gob.es/boloniaensecundaria/img/Declaracion_Bolonia.pdf

DECLARACIÓN DE LA SORBONA. (1998). Declaración de la Sorbona. Declaración conjunta para la armonización del diseño del Sistema de Educación Superior Europeo a cargo de los cuatro ministros representantes de Francia, Alemania, Italia y el Reino Unido. Disponible en http://www.ciccp.es/ImgWeb/ Sede\%20Nacional/Declaraciones\%20Bolonia_Ber/Declaraci\%C3\%B3n\%20de\%20 Praga.pdf

DEClARACIÓN DE PRAGA. (2001). Declaración de Praga. Hacia el Área de la Educación Superior Europea. Declaración del encuentro de los Ministros Europeos en funciones de la Educación Superior en Praga, 19 de mayo del 2001. Disponible en http://www.ciccp.es/ImgWeb/Sede\%20Nacional/Declaraciones\%20Bolonia _Ber/Declaraci\%C3\%B3n\%20de\%20Praga.pdf

ELIAS, M. (2010). Impact of the Bologna process on Spanish students' expectations. International Journal of Iberian Studies, 23(1), 53-62.

FONT-MAYOLAS, S. Y MASFERRER, L. (2010). Conocimientos y actitudes de estudiantes universitarios respecto al Espacio Europeo de Educación Superior. Revista de formación e Innovación Educativa Universitaria. , 3(2), 88-96

GONZÁLEZ, J. Y WAGENAAR, R. (2003). Tuning educational structures in Europe: final report. Bilbao: Universidad de Deusto.

IGLESIA, M. C. (2011). Adecuación del grado de desarrollo de la formación en competencias a la necesidad en el entorno laboral, según la opinión de los estudiantes. Revista complutense de educación, 22(1), 71-92.

MALLADO, J. A. Y PÉREZ-LÓPEZ, J. A. (1985). La enseñanza universitaria ante las demandas de los discentes (profesores, métodos y medios). Revista de Enseñanza Universitaria, 9, 61-80.

MENDEZ, R. (2008). Los jóvenes universitarios y su (des) orientación ante los nuevos retos que plantea el EEES. Educatio Siglo XXI, 26, 197 - 224. 
MEROÑO, A. L. Y RUIZ, C. (2006). Estrategias de adaptación al Espacio Europeo de Educación Superior a partir del conocimiento y actitud del profesorado universitario. Revista de Investigación Educativa, 24(1), 281 - 298.

MOLERO, D. (2007). Rendimiento académico y opinión sobre la docencia del alumnado participante en experiencias piloto de implantación del Espacio Europeo de Educación Superior. Relieve, 13(2), 175-190. Disponible en http://www.uv.es/RELIEVE/v13n2/ RELIEVEv13n2_2.html

MONTAÑO, J. J. Y PALOU, M. (2008). Estudio de la opinión del profesorado y de alumnado sobre la implantación de créditos europeos en la educación superior. Revista Electrónica Investigació Innovació Educativa i Socioeducativa, 1(0), 24 46. Disponible en http://www.in.uib.cat/pags/volumenes/vol1_num0/ oliver_montano/introduccion.html

REAL DECRETO 1125/2003, de 5 septiembre, por el que se establece el sistema europeo de créditos y el sistema de calificaciones en las titulaciones universitarias de carácter oficial y validez en todo el territorio nacional. Boletín Oficial del Estado núm.224, del 18 de septiembre de 2003.

REAL DECRETO 1393/2007, de 29 de octubre, por el que se establece la ordenación de las enseñanzas universitarias oficiales. Boletín Oficial del Estado núm.260, del 30 de octubre de 2007.

RUIZ, C., Y OLIVEROS, L. (2006). La opinión del alumnado de la Facultad de Educación (UCM) acerca de la metodología docente. Revista Complutense de Educación, 17(1), 29-48.

SÁEZ, F. J. (2000). La opinión de los estudiantes universitarios sobre el método docente de las facultades de ciencias. Revista de Investigación Educativa, 18(1), 157-182.

\section{Correspondencia con los autores}

Javier COTERÓN LÓPEZ

Facultad de Ciencias de la Actividad Física y del Deporte - INEF

C/Martín Fierro, $\mathrm{n}^{\circ} 7$

CP: 28040 Madrid

Tel.: 913364012

E-mail: j.coteron@upm.es

Evelia FRANCO ÁLVAREZ

Tel.: 679668635

E-mail: evelia.franco.alvarez@alumnos.upm.es

Javier GIL ARES

Tel.: 913364120

E-mail: javier.gil@upm.es 